\title{
Compter les morts
}

Document et fiction dans la frontière nord du Mexique (Sergio González Rodríguez, Roberto Bolaño)

Contar los muertos: documento y ficción en la frontera norte de México

\section{Melina Balcázar}

\section{(2) OpenEdition}

\section{Journals}

\section{Édition électronique}

URL : https://journals.openedition.org/agedor/5709

DOI : 10.4000 /agedor.5709

ISSN : 2104-3353

Éditeur

Laboratoire LISAA

Référence électronique

Melina Balcázar, "Compter les morts », L'Âge d'or [En ligne], 13 | 2020, mis en ligne le 01 octobre 2021, consulté le 14 octobre 2021. URL : http://journals.openedition.org/agedor/5709 ; DOI : https://doi.org/ 10.4000/agedor.5709

Ce document a été généré automatiquement le 14 octobre 2021.

L'Âge d'or. Images dans le monde ibérique et ibéricoaméricain 


\section{Compter les morts}

Document et fiction dans la frontière nord du Mexique (Sergio González Rodríguez, Roberto Bolaño)

Contar los muertos: documento y ficción en la frontera norte de México

\section{Melina Balcázar}

1 Afin d'introduire mon propos, je voudrais mettre en exergue deux images de l'artiste mexicaine Teresa Margolles :

- La première est celle d'une salle d'exposition vide, aucune œuvre, aucun objet, aucune trace ne sont visibles, mis à part celles de l'eau avec laquelle un homme nettoyant le sol ne cesse de l'humidifier. 
Salle d'exposition vide

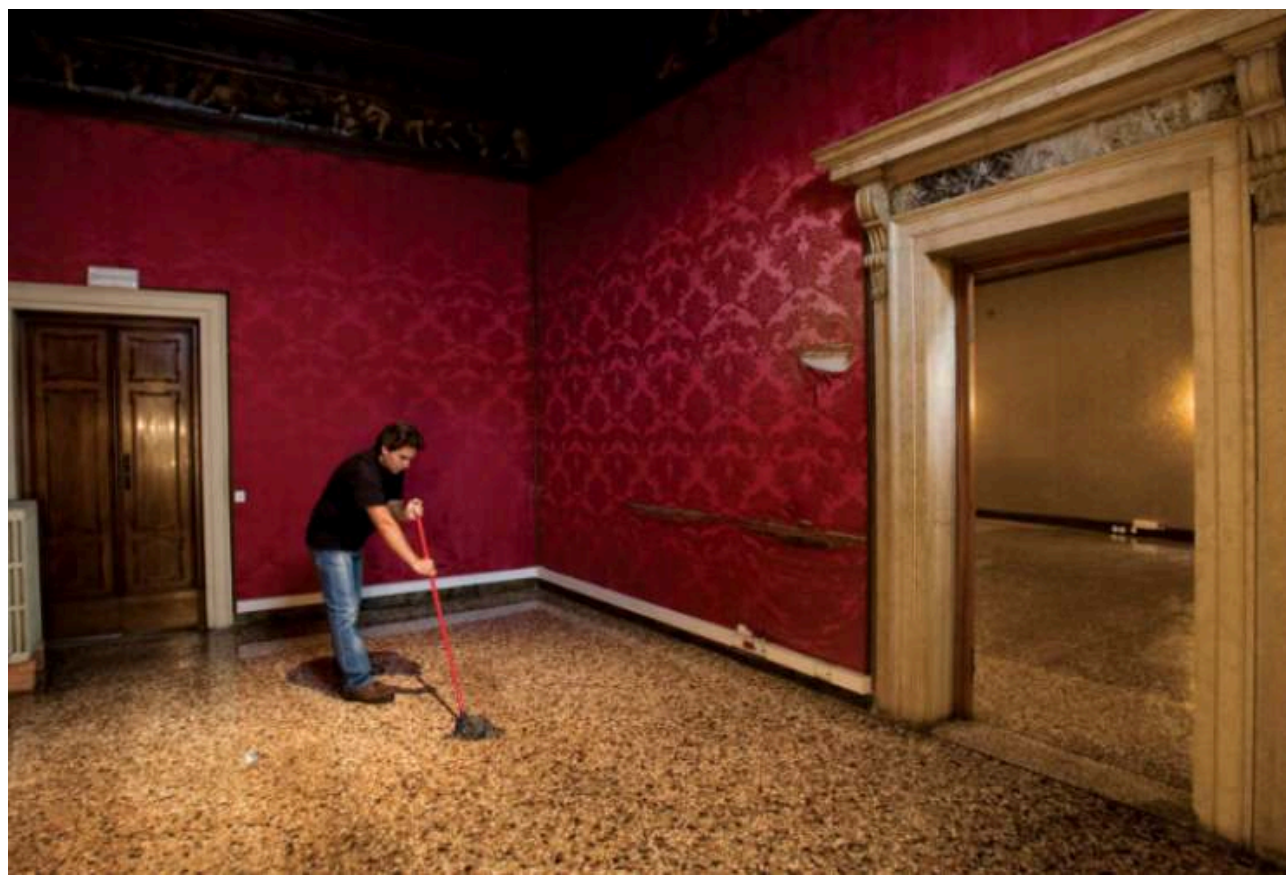

- La deuxième, celle d'une toile teintée de rouge, suspendue sur un mur, dans des moulures qui devaient autrefois encadrer des tableaux.

Toile teintée de rouge

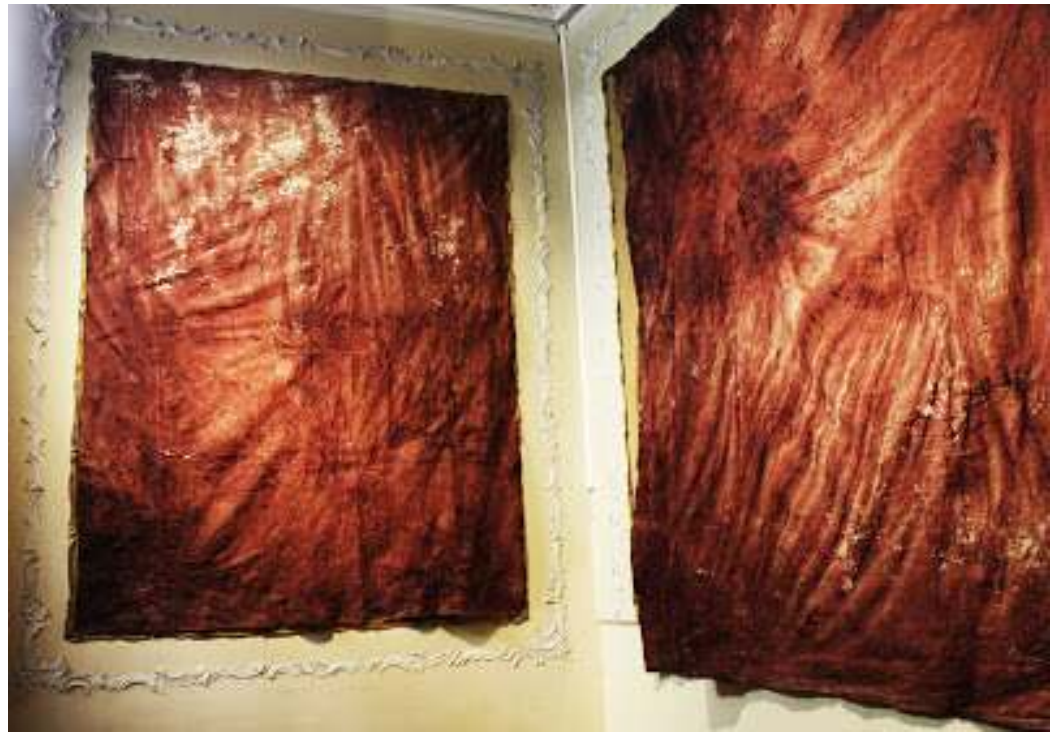

2 À la fin de leur parcours du palace vénitien accueillant le pavillon mexicain de la Biennale (2009), les visiteurs découvrent que les éléments employés pour ces deux images ont été prélevés sur des corps violemment tués dans les états de Sinaloa et de Basse Californie : le liquide de lavage est un mélange de sang et d'eau ayant servi à nettoyer leurs cadavres, et la personne qui procède à ce nettoyage est un membre de la famille ou un proche. Les visiteurs de l'exposition apprennent également que la toile, d'abord utilisée pour envelopper un cadavre, a été imbibée du sang de ces mêmes victimes. 
3 Le travail de Teresa Margolles rend ainsi la mort présente sans la montrer ni la représenter, elle la désigne par métonymie ${ }^{1}$. Car il ne s'agit pas seulement de dénoncer la violence - devenue une banalité et un phénomène social de plus - mais de lui donner une visibilité autre. Contrairement à la presse à sensation, elle ne sature pas l'espace de la salle d'exposition d'images explicites et privilégie le dépouillement, le résidu et l'indice au spectaculaire et à la surenchère. Par cette présence, devenue une hantise, elle crée d'abord chez le spectateur un malaise, qui frôle le dégoût, et le touche au sens propre comme au sens figuré. Est ainsi établie une forme de contact, par un élément organique qui a lui-même été en contact immédiat avec la mort: l'eau, le sang, les gestes répétitifs d'un proche du mort.

4 Dès le commencement de ma carrière, au début des années 90 , je travaille à une approche esthétique moins du thème de la mort que de celui des cadavres dans leurs différents aspects, y compris leurs implications socio-culturelles. Je travaille sur des corps sans vie, avec ce qui est en décadence, et je le fais toujours à partir de la même question : jusqu'où un cadavre peut-il endurer la souffrance? ${ }^{2}$

5 En effet, le travail de Teresa Margolles - outre la nécessaire dénonciation de la violence - ouvre vers une connaissance, pose les bases d'une « épistémologie de l'abject » ${ }^{3}$, celle du corps mort.

6 Une dernière précision : le titre de cette exposition, ¿De qué otra cosa podríamos hablar? [De quoi d'autre pourrions-nous parler?], me semble dire justement l'urgence à laquelle nous essayons de réfléchir ici : celle d'opposer à l'insoutenable de la violence, écriture, pensée, image. Car, en effet, que signifie écrire aujourd'hui dans un cadre où la mort violente constitue le quotidien de nos sociétés médiatisées ? Quelle position éthique et esthétique suppose d'écrire en étant littéralement entouré de morts?

7 C'est avec Sergio González Rodríguez que je souhaiterais commencer ce parcours dans le Nord du Mexique, par ce livre étonnant, inclassable de par son caractère hybride qu'est Des os dans le désert. Publié d'abord en Espagne en $2002^{4}$, il attire l'attention sur les assassinats de femmes dans la ville frontalière de Ciudad Juárez, dont les premiers registres datent de 1993. L'ouvrage commence avec des chiffres et des statistiques, mais surtout avec une énumération des victimes, comportant leurs noms, leur description physique et une narration minutieuse des sévices qu'elles ont subis. Dès les premières pages est entamé «un montage spectral de visages, de données, de caractéristiques physiques, de taches indiscernables $»^{5}$, comme une manière de "rassembler ce qui est éparpillé » afin de "contredire la "vérité" officielle » (p. 285). Le travail d'enquête de Sergio González Rodríguez, en tant que journaliste pour le quotidien national Reforma, constitue le point de départ du livre, composé de témoignages, investigations sur le terrain, consultation d'archives institutionnelles, en particulier policières et judiciaires, mais également des recherches historiques, et ce afin d'identifier les racines du «mal expansif» (p. 24) que cette ville frontalière condense :

Ciudad Juárez, ainsi nommée depuis 1888 et anciennement Paso del Norte («Passage du Nord»), était une mission à l'époque coloniale, puis un territoire d'immigration, de transit, de contrebande, et bien souvent de violence extrême. L'économie informelle ou clandestine tout comme la vie qui en découle généralement font partie de son histoire et de son développement. Mais dans la dernière moitié du $\mathrm{xx}^{\mathrm{e}}$ siècle, Ciudad Juárez a suivi les modèles multinationaux de production industrielle avec des technologies de pointe. Parallèlement, elle devenait de plus en plus importante en tant que territoire versé dans le trafic de drogue. (p. 46) 
C'est dans cette production en masse qui génère un excédant humain - «le principal problème, c'est la surpopulation $»^{6}$, nous dit l'auteur - que se trouverait l'origine des meurtres de femmes :

[...] être femme à Juárez implique de vivre "en tant que telle dans un système relationnel désavantageux, une ville et un espace qui les rendent vulnérables". Le milieu dans lequel les femmes évoluent ne connaît aucune politique de développement et s'érige sur un système de relations de pouvoir qui refuse de reconnaitre les formes d'asymétrie structurelle de la société. Elles constituent une véritable réserve et une usine de sous-traitance de main-d'œuvre. ${ }^{7}$

Dans des contextes de violence sociétale, le recours aux sources documentaires et à l'enquête s'impose pour recueillir des témoignages, faire entendre les voix des victimes autant que celles des bourreaux, et répondre à l'urgence de rendre compte de situations d'injustice, voire de corruption et d'assassinat. L'utilisation du document et des archives exige donc un autre mode d'écriture, ainsi que l'élaboration d'une nouvelle approche de la composition, susceptibles de «faire des associations et des rapprochements entre les événements que la vie quotidienne éparpille au milieu du chaos de l'information, au risque de provoquer une amnésie générale $»^{8}$.

Dans le livre de Sergio González Rodríguez, un type de document occupe une place privilégiée : les rapports de médecins légistes qui introduisent un mode de narration singulier en faisant parler leurs corps. Dans la partie finale du livre, intitulée «La vie interrompue ", sont recensés, du plus récent au plus ancien, les meurtres de 197 femmes, âgées de 7 à 30 ans. 197 fois sont rapportées les tortures qu'elles ont subies, les lieux sordides où elles ont été retrouvées, le moment où leurs vies ont été brisées :

23/09/02 : Erika Pérez, âgée de 25 à 30 ans ; cheveux châtains, chemisier avec des fleurs imprimées, pantalon et culotte baissés sur les genoux, courroie du sac autour du cou ; chemin de terre battue qui part du carrefour des rues Paseo del Río et Camino San Lorenzo.

23/09/02 : corps de femme non identifiée, ossements datant d'environ onze mois ; à côté, une culotte, un pantalon de couleur orange, des chaussettes blanches, 11 épingles à cheveux ; derrière la maquiladora $\mathrm{BRK}$, près des voies ferrées et de la voie Juan Gabriel.

28/08/02 : Dora Alicia Martínez Mendoza, 34 ans ; 35 blessures causées par un instrument pointu et tranchant sur tout le corps ; domiciliée au 6100 de la rue Pitaya y Cebada, Colonia el Granjero.

22/06/02 : corps de femme non identifiée ; lotissement Paso de los Virreyes ; balle dans la tête.

09/06/02 : Carmen Ivón Ontiveros Rodríguez, 13 ans ; à demi enterrée ; coups de marteau sur le crâne ; vraisemblablement violée ; arrière-cour d'une maison située à proximité de son domicile 9 .

11 Le médecin légiste est celui, nous dit Giovanni De Luna dans Le cadavre de l'ennemi. Violence et mort dans la guerre contemporaine (2006), qui fait " "parler les morts", interroge les cadavres afin de plonger dans ce qui a été leur vie, dans tout ce qui a constitué leur passé et qui est resté prisonnier dans leurs corps. $»^{10} \mathrm{Il}$ s'agit des « corpsdocument", narrés par le médecin légiste, qui leur restitue une individualité spécifique, en les dégageant de la masse informe dans laquelle la violence les a plongés. Des os dans le désert devient ainsi une archive de ce "matériau humain ${ }^{11}$ - pour 
reprendre le juste titre du livre de Rodrigo Rey Rosa -, une archive du réalisme extrême.

12 Sergio González Rodríguez ne cède donc pas à la tyrannie de l'immédiateté (l'autre sens de l'urgence que nous interrogeons ici) et prend le temps de nommer chacune de ces femmes, dans une éprouvante énumération, sorte de litanie pour conjurer l'oubli. D'où cette nécessité de dire leurs noms, de dénombrer chaque morte, d'éviter que ces cadavres se confondent avec une masse qui neutralise ce que la mort comporte de désignation singulière, de nomination et dénombrement. Car, sans le pouvoir de nommer, le langage se trouverait lui-même dépossédé de sa propre mémoire ${ }^{12}$.

13 Mais encore, que faire des corps de ces femmes sauvagement assassinées? Ne pas les ignorer comme l'ont fait les autorités mexicaines et les lire, semble être la réponse de Sergio González Rodríguez :

14 Le pays abrite maintenant un infâme ossuaire, visible malgré la complaisance des autorités. Ces crimes finissent par avoir des répercussions dans le monde entier. Me souvenir est devenu pour moi un impératif qu'il m'est parfois difficile de respecter [...]. «Tâche quand même de ne pas oublier, me dis-je. Tu fais partie des morts et des mortes, tu te prosternes devant eux. » Se souvenir, même si cela semble excessif, voir décalé à notre époque. Que d'autres sachent que je me souviens, qu'ils lisent ce qui est écrit en rouge pour comprendre ce qui est écrit en noir. ${ }^{13}$

Un excès de mémoire qui se traduit par une écriture qui déborde les limites de l'enquête journalistique en faisant recours à la narration, comme une manière de retrouver le fil de ces vies interrompues, de relier les lignes de cette écriture autre dont leurs corps portent la trace.

16 En reprenant le concept de nécropolitique du penseur camerounais Achille Mbembe ${ }^{14}$, qui place la mort et non la vie au cœur du politique, l'écrivaine mexicaine Cristina Rivera Garza propose de parler de "nécroécritures ", dans la mesure où le contexte de mort qui les fait surgir les force à remettre en question «l'état actuel de nos langages $»^{15}$, à travers leur devenir document, archive, fiche anamnestique :

Les écrits produits dans un contexte de nécropolitique sont, en réalité, des fiches anamnestiques de la culture. [...] Loin de leur «donner naissance», les écrivains agissent en médecins légistes, et lisent attentivement, interrogent, creusent ou exhument à travers le recyclage ou la copie, re-contextualisent, rendent sensible l'absence des disparus, leurs corps. À la fin, avec un peu de chance, ils enterrent ces cadavres dans le corps du lecteur, où [...] ils deviendront ces rêves qui jamais nous laisseront dormir en paix. Et si ceci n'est pas perturber radicalement nos perceptions et notre expérience du monde, ce serait quoi alors? ${ }^{16}$

17 Et c'est ce que semble avoir lu à son tour Roberto Bolaño, qui poursuit le récit anamnestique entamé par son ami Sergio González Rodríguez ${ }^{17}$. Dans son roman posthume $2666^{18}$, il assume cet excès, ce débordement de la mémoire, donnant lieu au récit éprouvant de 111 meurtres. Comme s'il suivait jusqu'à l'épuisement la tâche que González Rodríguez s'était imposée.

18 Ces corps, qui constituent la matière centrale du roman, livrent un témoignage provenant de l'intérieur même de la mort et qui nous parvient à travers les rapports des médecins légistes, dont le langage technique est repris pour raconter ces crimes. Rien n'est donc dit de manière voilée. Aucun détail ne nous est épargné: les mutilations, les traces des moyens employés pour les torturer et les violer sont redits encore et encore, comme si seule la démesure de cette répétition pouvait rendre justice 
aux victimes. Ces descriptions minutieuses mettent en évidence la réalité de la souffrance dans la torture : assassinées par strangulation - dont « la fracture de l'os hyoïde » devient une signature -, poignardées ou battues jusqu'à la défiguration, violées par « les trois voies ", jetées dans des décharges, dans des terrains vagues, dans le désert. Au-delà de l'enquête menée pour découvrir l'identité de l'assassin ou des assassins, Bolaño décide d'inclure aussi, à l'instar de Sergio González Rodríguez, dans sa longue énumération de femmes assassinées, les victimes de violences conjugales et familiales. Parmi les crimes les plus choquants se trouvent ceux qui ont été commis par des proches, qui les ont empalées ${ }^{19}$, brûlées ${ }^{20}$, mutilées ${ }^{21}$.

En décrivant le supplice des femmes, l'auteur cherche plutôt à situer son récit du côté des victimes dont le corps meurtri constitue le seul témoignage disponible. Il restitue ici encore une individualité spécifique à chacune de ces femmes qui ont été dépossédées de leur humanité. En niant leur identité, soit de manière délibérée - dans les cas de meurtriers -, soit par incompétence - dans celui des autorités - leurs dépouilles sont le plus souvent destinées à un dernier outrage : celui du refus d'une tombe digne. Retrouvées parmi les poubelles - certaines dans des sacs en plastique -, nombre d'entre elles finissent "balancées sans attendre davantage dans la fosse commune $\aleph^{22}$ ou dans le dépôt des cadavres de l'école de Médecine ${ }^{23}$. Les différentes attitudes devant ces cadavres - allant de l'indifférence des policiers à l'extrême familiarité des ambulanciers qui mangent des tacos à proximité, jusqu'aux larmes et prières de certains habitants - mettent en évidence leur solitude, leur «abandon [desamparo], quelque chose de très latino-américain ${ }^{24}$.

20 Car les mortes de Santa Teresa - comme celles de Ciudad Juárez pour Sergio González Rodríguez - sont les victimes de ce mal en expansion, global, qui est celui de la production en masse, étroitement liée à l'anéantissement de l'humain, et dont Bolaño retrace la généalogie tout au long de son œuvre : l'extermination nazie de masse et son retour, comme une hallucination, en Amérique latine au moment des dictatures, aboutissent aux meurtres en série de femmes au Nord du Mexique. Avec cet humour noir qui le caractérisait, Bolaño donne à l'un de trois médecins légistes de Santa Teresa le surnom du «docteur Mengele de Sonora $»^{25}$. Il semble trouver dans l'Allemagne nazie non seulement le modèle de la destruction en masse des populations entières mais, surtout, la possibilité pour un État de faire de la mort un projet. Chez Bolaño, mort et pouvoir deviennent, de fait, indissociables. Dans 2666, ce lien est mis en évidence dans de nombreux passages, à travers l'assimilation des cadavres des femmes avec des déchets industriels : "Dans la décharge où l'on trouva la morte s'entassaient non seulement les détritus des habitants des taudis mais aussi les déchets de toutes les maquiladoras. $\gg 26$

21 La violence de l'antisémitisme et la violence de la misogynie sont ainsi à l'origine d'une horreur qui associe la production et le meurtre en série à la liturgie du sacrifice. La signification sacrificielle, véhiculée par le mot "holocausto", utilisé en espagnol pour désigner la Shoah, est rendue sensible par des passages comme celui décrivant la plus ancienne maquiladora de Santa Teresa : « la maquiladora EMSA [...] n'était dans aucune zone industrielle mais au beau milieu de la colonia La Preciada, telle une pyramide de couleur melon, avec son autel de sacrifices caché derrière les cheminées ${ }^{27}$

22 La référence à la pyramide n'est pas sans rappeler ici les sacrifices rituels des Aztèques qui sont par ailleurs longuement évoqués dans "La partie d'Archimboldi" ${ }^{28}$. Mais le recours de Bolaño à cette référence historique est avant tout critique, comme une 
manière de contester toute mythification de la mort. Bien que l'explication des crimes par un retour de cette violence ancestrale, qui serait latente dans le Mexique actuel, paraisse être en effet une des pistes avancées par le roman, le choix narratif de Bolaño n'est pas, comme cela a été affirmé, celui de prolonger « une ancienne et équivoque tradition ou discours représentant le Mexique comme un pays lointain et exotique où ont été conservées des forces naturelles primitives ou le pays où habitent des êtres incarnant l'esprit maléfique et criminel aztèque ${ }^{29}$. Il fait plutôt cette critique en utilisant son arme de prédilection: l'humour. Et l'une de ses cibles est, à nouveau, Octavio Paz, dont la conception de l'histoire comme retour - «vuelta» - est remise en question par une lecture parodique. Sergio González Rodríguez, devenu un personnage du roman, expose ainsi à un ancien chef de la police de Mexico, le général Humberto Paredes, l'hypothèse des tournages de snuffmovies à Santa Teresa, dont les protagonistes seraient les femmes assassinées :

Le général ne fut pas d'accord: d'après lui, la pornographie avait atteint son plein développement peu avant la Révolution française. Tout ce qu'on pourrait voir dans un film hollandais actuel ou une série de photos ou dans un opuscule licencieux avait déjà été fixé avant 1789 , et dans une grande mesure était une répétition, un tour d'écrou à un regard qui regardait déjà. Général, lui dit Macario López Santos [journaliste de la section faits divers], parfois vous parlez vraiment comme Octavio Paz, vous ne seriez pas en train de le lire? Le général lâcha un grand rire et dit que la seule chose qu'il ait lue, et il y avait de ça des années, c'était Le Labyrinthe de la solitude, et qu'il n'y avait rien compris. ${ }^{30}$

Dans un autre passage, c'est le cliché d'un Mexique où vie et mort se confondent qui est visé :

Vous voulez dire que vous croyez que Kelly est morte, lui ai-je crié. Plus ou moins, a-t-il dit [...]. Comment ça, plus ou moins? ai-je crié. On est mort ou on n'est pas mort putain de Dieu! Au Mexique, on peut être plus ou moins mort, m'a-t-il répondu très sérieusement. Je l'ai regardé avec l'envie de le gifler. [...] Non, [...] ni au Mexique ni nulle part ailleurs sur la planète quelqu'un peut être plus ou moins mort. Arrêtez de parler comme un guide touristique. [...] Loya a souri. De quoi riezvous ? lui ai-je demandé. J'ai trouvé amusant le truc du guide touristique, a-t-il dit. J'en ai marre des Mexicains qui parlent et se comportent comme si tout ceci était Pedro Páramo, lui ai-je dit. ${ }^{31}$

Bolaño casse par ce recours à l'humour l'effet hypnotique de l'énumération, de la douloureuse accumulation des noms entreprise par Sergio González Rodríguez et permet de tenir à distance le pathos et l'émotion pour rester alerte. Puisque, ce qu'il veut creuser, c'est la réalité de la mort dans une ville à la frontière entre le Nord et le Sud, la manière dont l'excédent humain y devient cadavre, pur déchet, comme le montrent les passages - très crus - qu'il consacre à la décharge El Chile ${ }^{32}$. C'est face à l'horreur de la violence contemporaine que «La partie des crimes » nous place, comme face à une tête de Méduse : cette violence globale contre les plus démunis rend leur corps impossible à regarder, puisqu'elle le défigure et lui fait perdre sa forme humaine $^{33}$. Une violence qui, comme le signale Adriana Cavarero dans Horrorism. Naming contemporary violence, ne se contente pas de tuer, car tuer serait encore trop peu par rapport à l'objectif à atteindre qui est celui de détruire « le caractère unique du corps, en en déchirant la vulnérabilité constitutive. Ce qui est en jeu n'est pas la fin de la vie humaine mais de la condition humaine elle-même, telle qu'elle s'incarne dans la singularité des corps vulnérables. $»^{34}$ au risque de la reproduire à son tour, de s'en faire l'écho. Mais, comme il tenait à 
l'affirmer, la tâche de l'écrivain consiste à « savoir mettre la tête dans l'obscur, savoir sauter dans le vide. [...] Courir au bord du précipice : d'un côté l'abîme sans fond, et de l'autre, les visages que l'on aime, les visages souriants que l'on aime, et les livres et les amis et les repas. $\|^{35}$ En effet, la mort est toujours à proximité dans le monde actuel, comme le montrait déjà Bolaño dans Étoile distante, et celui qui veut écrire ne doit pas l'ignorer :

L'atelier de Soto se trouvait, je n'ai jamais su pourquoi, dans la faculté de médecine, c'était une pièce mal meublée et mal ventilée, qu'un couloir à peine séparait de l'amphithéâtre où les étudiants débitaient des cadavres en morceaux pendant les cours d'anatomie. L'amphithéâtre, évidemment, sentait le formol. Le couloir puait aussi, parfois. Et certaines nuits, car l'atelier de Soto fonctionnait tous les vendredis de huit à dix, quoique le plus souvent il prît fin à minuit passé, la pièce s'imprégnait de cette odeur de formol que nous essayions vainement de masquer en allumant cigarette sur cigarette. ${ }^{36}$

Mais enfin, «il n'y a pas que de la mort dans la mort $»^{37}$. Il y a aussi «créativité $»^{38}$, " imagination " ${ }^{39}$, « avenir », comme nous le rappelle John Berger dans ses Douze thèses sur l'économie des morts: "Quelle relation entretiennent morts et vivants avec ce qui n'est pas encore arrivé, avec l'avenir. Tout l'avenir est la construction dans laquelle leur "imagination" est investie. $»^{40}$

Compter les morts participe bien de cette économie, d'où sa teneur politique, puisque cet acte remet en question la séparation entre morts et vivants, manière de questionner, brouiller les régimes de visibilité dominants, la place que les sociétés contemporaines accordent à ceux qui ont disparu et hantent, par leur matérialité spectrale, l'espace public :

Comment coexistent, cohabitent les vivants avec les morts? Jusqu'à la déshumanisation de la société dans le capitalisme, tous les vivants envisageaient l'expérience des morts. C'était leur ultime avenir. Par eux-mêmes, les vivants étaient incomplets. Ainsi, les vivants et les morts étaient interdépendants. Sans cesse. Seule une forme unique d'égotisme a rompu cette interdépendance. Avec des résultats désastreux pour les vivants, qui pensent à présent les morts comme éliminés. ${ }^{41}$

D'où la nécessité, parfois impérieuse, de donner une visibilité autre aux cadavres qui sont - comme le souligne Teresa Margolles - un «thermomètre social» permettant d'« analyser ce qui se passe dans les sociétés ${ }^{42}$.

Pour conclure, peut-être devrais-je évoquer une autre manière de compter les morts, qui me semble se rapprocher de la démarche de Sergio González Rodríguez et qui, on peut supposer, aurait intéressé Roberto Bolaño. Il s'agit de la carte de féminicides qu'une habitante d'une banlieue défavorisée de Mexico a créée en 2015: https:// feminicidiosmx.crowdmap.com/. Confrontée au manque d'informations fiables sur le nombre de femmes assassinées dans le pays - aucune organisation gouvernementale n'a jamais été en mesure de les fournir -, María Salguero a commencé à consacrer son temps libre, en dehors de ses études de géophysique et son travail, à leur recensement. Elle a trouvé ses sources principalement dans les journaux, en particulier ceux consacrés aux faits divers (comme $\mathrm{El} \mathrm{Gráfico} \mathrm{o} \mathrm{Alarma).} \mathrm{En} \mathrm{recoupant} \mathrm{les} \mathrm{informations,}$ elle est arrivée à constituer une base de données qui a même mis en difficulté la parole officielle, qui affirmait inlassablement une baisse significative de féminicides pendant le dernier gouvernement. María Salguero accorde une grande attention à la partie narrative contenue dans ces journaux à sensation, puisqu'ils offrent de nombreux détails pris sur la scène du crime. 
Ce qui fait écho à cette intuition qui a guidé Sergio González Rodríguez lors de l'écriture de Des os dans le désert :

La lecture de la presse me donne des idées noires et me fait songer à l'une des pensées les plus vraies et les plus inquiétantes de Leonardo Sciascia, quand il affirme que c'est dans les journaux que s'étale toute la réalité, même si elle apparaît couverte d'un voile qui laisse malgré tout entrevoir ce qui se passe. Par conséquent, il faut lire entre les lignes et ne pas oublier. Ceux qui ont le pouvoir veulent aussi avoir le monopole de la vérité. ${ }^{43}$

\section{NOTES}

1. Caroline Perrée, «Au Mexique, la mort suinte dans l'art. Teresa Margolles : quand l'œuvre saigne », Amerika [En ligne], 8 | 2013, mis en ligne le 22 avril 2014, consulté le 06 novembre 2018. URL : http://journals.openedition.org/amerika/3812 ; DOI : 10.4000/amerika.3812

2. Teresa Margolles citée dans Norma Lazo, Las siete virtudes contemporáneas, México, Fondo editorial del Estado de México, 2016, p. 164.

3. Cuauhtémoc Medina parle d'une "estética de lo ominoso", au sens de l'unheimlich freudien ("Espectralidad materialista", catalogue de l'exposition ¿De qué otra cosa podríamos hablar?).

4. Il faudra attendre 2006 pour qu'une édition mexicaine soit possible, puisque comme le lui fait savoir son éditeur « il y avait un veto » contre le livre.

5. Sergio González Rodríguez, Des os dans le désert, trad. Isabelle Guignon, Passage du Nord/Ouest, Albi, 2007, p. 159 (traduction modifiée).

6. Ibid., p. 42.

7. Ibid., p. 49.

8. Ibid., p. 285.

9. Ibid., p. 260.

10. Giovanni De Luna, El cadáver del enemigo. Violencia y muerte en la guerra contemporánea, trad. Patricia Orts, Madrid, 451 Editores, 2007, p. 38.

11. Rodrigo Rey Rosa, Le Matériau humain, trad. Gersende Camenen, Paris, Gallimard, 2016, coll. Du monde entier.

12. Pierre Fédida, « Compter les morts », L'inactuel, nº 1, 1994.

13. Sergio González Rodríguez, Des os dans le désert, op. cit., p. 289 (traduction modifiée).

14. Mbembe Achille, "Nécropolitique ", Raisons politiques, trad. Émilie Cousin, Sandrine Lefranc, Eleni Varikas, 2006/1 ( $\mathrm{n}^{\circ} 21$ ), p. 29-60. DOI : 10.3917/rai.021.0029. URL : https://www.cairn.info/ revue-raisons-politiques-2006-1-page-29.htm

15. Cristina Rivera Garza, Los muertos indóciles, Mexico, Tusquets, 2013, p. 33.

16. Ibid., p. 38-39.

17. Sergio González Rodríguez, « Roberto Bolaño, détective et artiste », Europe, numéro consacré à Roberto Bolaño, dirigé par Anne Picard et Melina Balcázar, n 1070-1071-1072, juin/juil./août 2018.

18. Roberto Bolaño, 2666, trad. Robert Amutio, Paris, Christian Bourgois, 2008.

19. Ibid., p. 448-450.

20. Ibid., p. 486-488.

21. Ibid., p. 526-528.

22. Ibid., p. 593. 
23. Ibid., p. 484.

24. Ibid., p. 601.

25. Ibid., p. 571.

26. Ibid., p. 411.

27. Ibid., p. 515.

28. Ibid., p. 790-794.

29. Fernando Saucedo Lastra, México en la obra de Roberto Bolaño, Madrid/Mexico, Iberoamericana/ Bonilla editores, 2015, p. 12. Une interprétation similaire est adoptée par Caroline Perrée, «Au Mexique, la mort suinte dans l'art. Teresa Margolles : quand l'œuvre saigne ", Amerika, op. cit. : «la violence au Mexique n'est pas une nouveauté générée par la politique d'éradication des cartels de l'ancien président Felipe Calderón. Elle est, à bien des égards, une constante dans la construction identitaire du pays. La violence sur le corps et la nécessité de faire couler le sang remontent aux temps préhispaniques, lorsque les Aztèques sacrifiaient leurs victimes à des fins cultuelles. »

30. Roberto Bolaño, 2666, op. cit., p. 610-611.

31. Ibid., p. 710.

32. Ibid., p. 426, 483-484, 684-685.

33. Roberto Bolaño, Étoile distante, trad. Robert Amutio, Paris, Christian Bourgois, coll. « Titres ", 2002, p. 22.

34. Adriana Cavarero, Horrorism. Naming contemporary violence, trad. William McCuaig, New York, Columbia University Press, 2009, p. 8.

35. Roberto Bolaño, Entre parenthèses, trad. Robert Amutio, Paris, Christian Bourgois, 2011, p. 46.

36. Roberto Bolaño, Étoile distante, op. cit., p. 22.

37. Pascal Quignard, Requiem. Enfant qui repose et roche de Cumes par Leonardo Cremonini, Paris, Galilée, 2006, p. 59.

38. John Berger, "Thèse 4" dans "Twelve Theses on the Economy of the Dead", Pages of the Wound: Poems, Drawings, Photographs, 1956-96, Londres, Bloomsbury Publishing, 1996.

39. Ibid., "Thèse 10".

40. Ibid., "Thèse 11".

41. Ibid., "Thèse 12".

42. "Arte de entre los muertos", entretien avec Teresa Margolles, El País, 24 juin 2012, consulté le 06 novembre 2018. URL : https://elpais.com/cultura/2012/06/24/actualidad/ 1340529212_935079.html

43. Sergio González Rodríguez, Des os dans le désert, op. cit., p. 285.

\section{RÉSUMÉS}

La mort violente, le travail précaire, la pauvreté font partie du quotidien de la frontière nord du Mexique. C'est à cette mort effroyable, car brutale et quotidienne, que l'écriture contemporaine se trouve confrontée et qui la porte à trouver d'autres dialogues esthétiques et éthiques. Devant cette réalité, la tâche de l'écrivain devrait être, selon l'injonction lancée par l'écrivain chilien Roberto Bolaño, de "savoir mettre la tête dans l'obscur, savoir sauter dans le vide " et écrire minutieusement l'horreur en intégrant document et fiction. À partir des exemples de Roberto Bolaño et Sergio González Rodríguez - qui ont été profondément marqués par cette Frontière qui 
sépare le Nord du Sud, les riches des pauvres, les hommes des femmes, l'anglais de l'espagnol -, on analyse ici la portée politique de cet acte, obsessionnel et douloureux, de «compter les morts ».

La muerte violenta, el trabajo precario, la pobreza son el día a día en la frontera norte mexicana. La escritura contemporánea se confronta sin cesar al horror de la muerte, brutal y cotidiana, conduciéndola así a crear otros diálogos estéticos y éticos. Ante tal realidad, el deber del escritor debería consistir, según las palabras de Roberto Bolaño, en "saber meter la cabeza en lo oscuro, saber saltar al vacío, saber que la literatura básicamente es un oficio peligroso" y escribir minuciosamente el horror, recurriendo al mismo tiempo al documento y la ficción. A partir de los ejemplos de Roberto Bolaño y Sergio González Rodríguez - profundamente marcados por la Frontera que separa el Norte y Sur, ricos y pobres, hombres y mujeres, inglés y español - se analiza aquí las implicaciones políticas del acto obsesivo y doloroso de "contar a los muertos".

INDEX

Mots-clés : féminicide, frontière, violence, mort, Bolaño (Roberto), González Rodríguez (Sergio) Palabras claves : femenicidio, frontera, violencia, muerte, Bolaño (Roberto), González Rodríguez (Sergio)

\section{AUTEUR}

MELINA BALCÁZAR

Colegio de México 